\title{
RESONATORS WITH INTRACAVITY PHOTONIC CRYSTALS
}

\author{
M. Peckus ${ }^{\text {a, b }}$, R. Rogalskis ${ }^{\text {a }}$, V. Sirutkaitis ${ }^{a}$, and K. Staliūnas ${ }^{c, d}$ \\ ${ }^{a}$ Laser Research Center, Vilnius University, Sauletekio 10, LT-10222 Vilnius, Lithuania \\ E-mail: martynas.peckus@ff.vu.lt \\ ${ }^{\mathrm{b}}$ Center for Physical Sciences and Technology, Savanoriu 231, LT-02300, Vilnius, Lithuania \\ 'Departament de Fisica i Enginyeria Nuclear, Universitat Politècnica de Catalunya, Colom 11, 08222 Terrassa, \\ Barcelona, Spain \\ d Institució Catalana de Reserca i Estudis Avançats (ICREA), Pg. Lluis Companys, 23, 08010 Barcelona, Spain
}

Received 4 December 2012; accepted 20 December 2012

\begin{abstract}
We investigate theoretically and experimentally light dynamics in plane-mirror Fabry-Pérot resonators filled with two- and three-dimensional photonic crystals. It has been predicted that the diffraction of such resonators can be manipulated [20] and used to control the linear and nonlinear light pattern formation there. Here we study the phenomenon in detail. We show the hyperbolic shape angular transmission profiles in case of a two-dimensional photonic structure (obtained by the one-dimensional modulation of the surface of the mirrors) and study the sub- and superdiffractive regimes in such resonators. We also summarize and review the previous results [21] of a resonator filled by a three-dimensional photonic structure (obtained by the twodimensional modulation of the mirrors).
\end{abstract}

Keywords: photonic crystals, resonators, diffraction

PACS: 42.55.Tv, 42.25.Fx, 42.79._e

\section{Introduction}

Pattern formation in nonlinear optical resonators is a fascinating subject, a systematic study of which started more than two decades ago [1]. First of all, the pattern formation in nonlinear optical resonators resembles spontaneous pattern formation in other systems in the nature and technology [2]. The appearance of structures, seemingly from nothing, is a subject which fascinates not only physicists, but everybody interested in "philosophical" problems of self-evolution in the nature [ 3 ]. The nonlinear optical systems are nice and well controllable playground for studying these building bricks of evolution in the nature. On the other hand, the patterns in optics are potentially applicable to digital and analogous information processing.

After very active 1990s, there arrived a decade of moderate interest in nonlinear spatial patterns. This is perhaps due to the fact that nonlinear light patterns did not enjoy quick applications from the very beginning. One reason for the lack of applications is that the "conventional" pattern forming nonlinear systems are relatively large in extent, and the basic localized patterns to be used in applications like optical vortices, solitons are of relatively large transverse dimensions. Therefore, in spite of a potentially huge speed of possible information processing (much larger than in conventional microelectronics), the localized light structures were supposed to be relatively large, i. e. of the size of the wavelength. Simply speaking, the photons of visible light are "large", much larger than electrons, and nonlinear formations from photons are large too. In spite of a potentially huge speed of information processing, the optical systems could not beat the microelectronic information processing systems, due to large dimensions of photons.

In recent years a revival of interest on nonlinear light patterns or on patterns in other similar 
systems can be seen. First of all, the structures in Bose condensates (vortices, etc.) are very similar to those discovered in the 1990s in nonlinear optics [4-8]. The Gross-Pitaevskii equation for zero-temperature Bose condensates is in fact the same nonlinear Schroedinger equation in nonlinear optics. Second, the huge growth of the interest in the field of surface plasmon polaritons [9-11] revived the pattern formation theory developed in nonlinear optics. The ensembles of polaritons are described by nearly the same order parameter equation as "pure" photons in optics. The polaritons (coupled states of a photon and surface plasmon) are in fact much "smaller" than pure photons; therefore, their applications in micro- and nano-optics become very promising.

In addition to the above-mentioned relations with other fields of nonlinear wave dynamics, the recent advance of microtechnologies allows building the linear and nonlinear microresonators and promises new possibilities for pattern formation in small compact nonlinear optical systems. In particular, wave diffraction in resonators becomes more controllable due to possibilities to fill the resonators with some exotic metamaterials [12]. Specifically, the negative-index metamaterials can invert the sign of diffraction, thus can allow nonevanescent propagation of subwavelength scale patterns. Another possibility is to fill the resonator with photonic crystals (PhCs). The latter idea also allows compensating positive diffraction of free space by negative diffraction of PhCs, thus also offers possibilities to reduce the spatial scale of the patterns. The idea is illustrated in Fig. 1. Recently, several concrete architectures have been proposed for such linear [13] and nonlinear [14, 15] resonators, allowing manipulation of resonator diffraction and

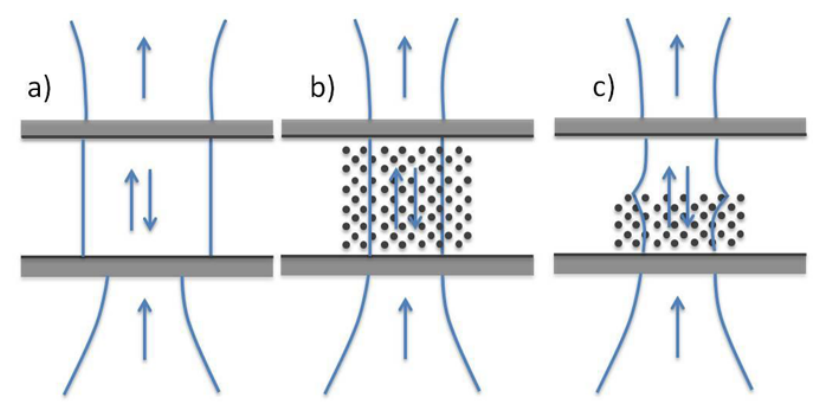

Fig. 1. Illustration of the beam inside a homogeneous resonator (a), and resonator completely (b) and partially (c) filled with PhCs. thus manipulating the scale of nonlinear patterns there. An experimental realization of the latter idea (filling the microresonator with PhCs) is behind the subject of the present article.

In spite of advancing technologies of microstructuring the matter, it is still difficult to build the microresonators fully or partially filled with $\mathrm{PhCs}$, as illustrated in Fig. 1. We follow a different technique, a different idea of realization, as illustrated in Fig. 2. a)

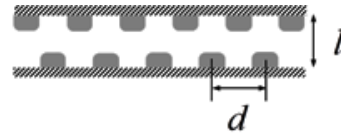

b)

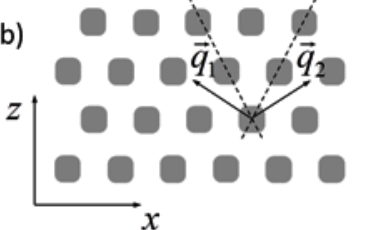

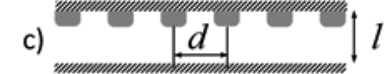

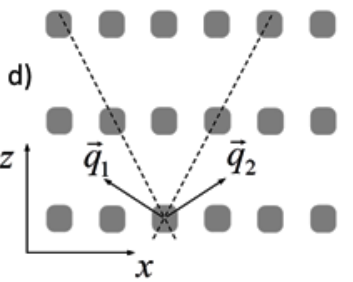

Fig. 2. Illustration of unfolded structure of the resonator: $(\mathrm{a}, \mathrm{b})$ double modulated mirror resonator, $(\mathrm{c}, \mathrm{d})$ single modulated mirror resonator.

Instead of directly filling the resonator (or part of it) with PhCs, we take advantage of the fact that the light wave travels periodically along the resonator. Therefore, it is sufficient just to place one period (one plane or one row) of $\mathrm{PhCs}$ in the resonator, and the resonating wave will behave in a very similar way as propagating along the photonic crystal. Technologically it is more convenient to modulate periodically a surface of one or both mirrors. Then the unfolded resonator results in a periodic structure. In other words, the photon making roundtrips along the resonator will behave in a similar way as propagating along the unfolded, periodic structure. Obviously, the behaviour of the light wave in the resonator is not completely equivalent to the behaviour of light in the unfolded structure, as the longitudinal mode structure is affected by unfolding. However, concerning the spatial propagation of the beams and of light patterns (i. e. concerning the transverse mode structure) the equivalence is complete. Therefore, if the unfolded $\mathrm{PhC}$ is of geometry corresponding to the nondiffractive (selfcollimating) regime [16, 17], then the photon resonating inside the "folded" structure will not show 
diffractive spreading either. If light propagating along the unfolded $\mathrm{PhC}$ shows angular (spatial) filtering [18, 19], then the light in the resonator can also be expected to show spatial filtering.

The above-described idea was proposed [20] and further elaborated and experimentally demonstrated [21]. The first fabricated mirrors were of a large period structure (with the period ten times larger than the wavelength); therefore, the numerical aperture of manipulated radiation was very small [20]. Developing technologies allow decreasing the periods of modulation, bringing the idea closer to reality. Now, sub-wavelength reflection gratings also become possible, which might open new perspectives for manipulating the waves even on a sub-wavelength scale.

The paper is devoted to the theoretical and experimental study of diffraction of the resonator filled with PhCs. The paper is organized as follows: Sec. 2 gives a short description of fabrication and of experimental measuring techniques. Sec. 3 reviews a transverse and longitudinal mode theory of modulated resonators, highlighting what differences could be expected compared with the nonmodulated case. Sec. 4 contains the derivation of the theoretical model used for numerical calculations. Original results are summarized in Sec. 5, where the subdiffractive and superdiffractive light dynamics in resonators is analysed numerically and experimentally. This study restricts to one-dimensionally modulated structures. For completeness, the results of two-dimensional mirrors are also presented in Sec. 6, which actually reviews the already published results [20, 21]. The paper ends with conclusions in Sec. 7. A short review of the relevant references on pattern formation on nonlinear optics is given in the Appendix.

\section{Fabrication and experiments}

The idea for such a resonator with an intracavity $\mathrm{PhC}$ is illustrated in Fig. 2: instead of creating modulation of the refraction index in both longitudinal and transverse directions of the intraresonator media, we modulated the surfaces of the mirrors. The mirrors, as shown in Fig. 2(a), are shifted with respect to one another by half of the modulation period. This, in an unfolded structure of such a resonator (Fig. 2(b)), results in a $2 \mathrm{D} \mathrm{PhC:} \mathrm{the} \mathrm{wave}$ resonating along the optical axis of the resonator is experiencing the periodic index of refraction both in longitudinal and transverse directions. The lateral shift of the mirrors results in the propagation along the diagonals of the rhombi of the unfolded lattice of the refraction index in the case of modulation in one transverse direction. We note that such geometry (propagation along the diagonals of a square lattice) is suitable for self-collimation [17, 22, 23] and for respective manipulation of diffraction. This is the reason why in the previous work [20, 21] such a geometry (in particular the laterally shifted mirrors) was chosen. Here, we show that it is also possible to use a single modulated mirror (Fig. 2(c)). The unfolded structure of this type of resonator has a square type (Fig. 2(d)).

The mirrors of the resonator used in experiments are shown in Fig. 3 for the cases of modulation in one and in two transverse dimensions. For the fabrication of the mirrors, the low roughness $(\lambda / 20)$ substrates were used. The working side of a mirror
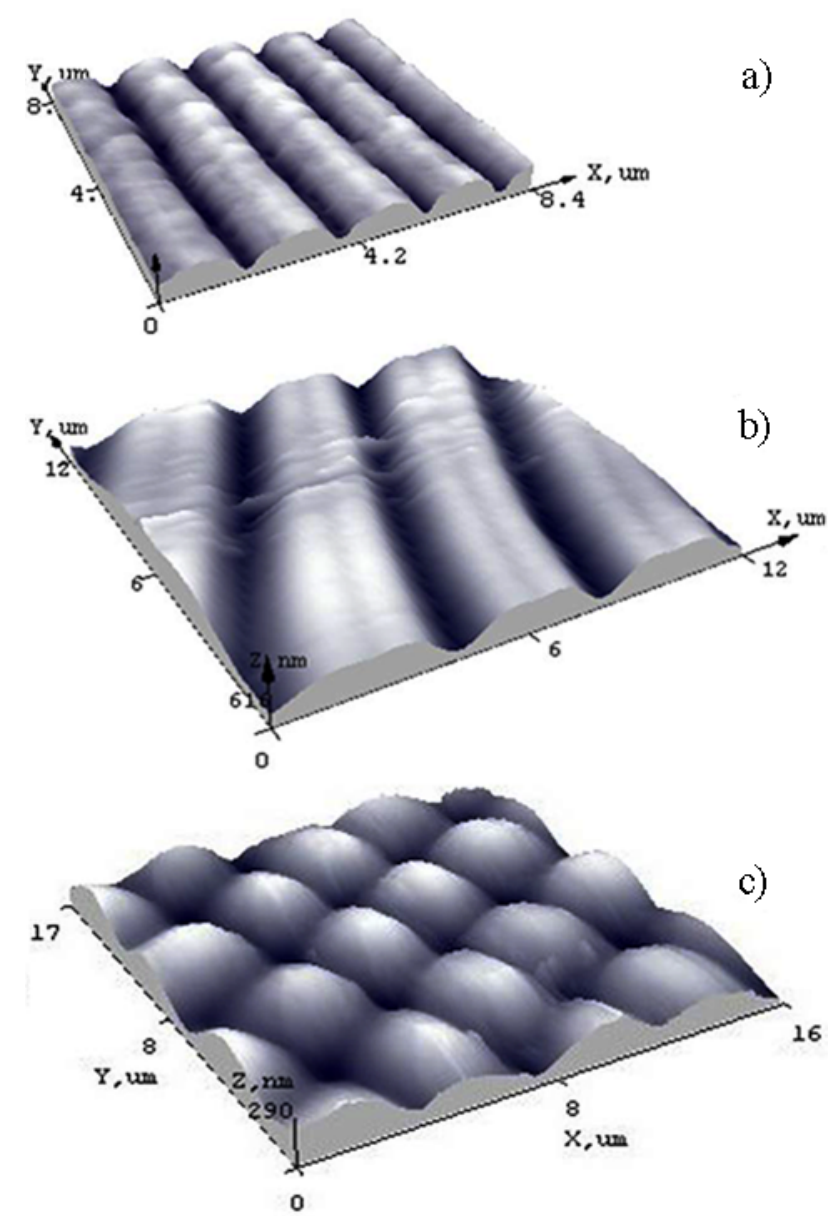

Fig. 3. AFM images of modulated surfaces of mirrors used in our experiments, where modulation periods are: (a) $\mathrm{d}_{\mathrm{x}}=2 \mu \mathrm{m}$, (b) $\mathrm{d}_{\mathrm{x}}=4 \mu \mathrm{m}$, and (c) $\mathrm{d}_{\mathrm{x}}=\mathrm{d}_{\mathrm{y}}=4 \mu \mathrm{m}$. 
was covered by high reflection (98.5\%) coatings, and the other side with antireflection. The modulation of the surface of the mirror was achieved by the photolithography technology. The surfaces of the mirrors were first covered with a thin film of the MICROPOSIT ${ }^{\circ}$ S $1805^{\circ}$ photoresist $(\sim 0.3 \mu \mathrm{m}$ thickness, $n=1.58$ index of refraction) using the spin coating technique. Next, using the contact photolithography technology, 1D periodic structure was exposed and etched on the layers of the film of the photoresist with the period $d=2 \mu \mathrm{m}$ and $d=4 \mu \mathrm{m}$ in the $1 \mathrm{D}$ modulation case. In the $2 \mathrm{D}$ modulation case, a film of a resist was exposed twice with a $90^{\circ}$ rotated template. In this way $d_{x}=d_{y}=4 \mu \mathrm{m}$ modulation was produced. The width of the etched groves was approximately half their period $\sim d / 2$, so the coatings on the mirror acted as the phase grating. Subsequently, the mirrors were heated in order to smoothen the profile of the photoresist and to make the surface modulation as close as possible to the harmonic one (see Fig. 2(a-c)).

Precise optomechanics or/and 3D piezo translator were used for cavity alignment and attenuation. The distance between the mirrors (linear length of the resonator) was varied in the range $l=5-40 \mu \mathrm{m}$ in accordance with the calculations (presented below). The mirrors (in the two modulated mirrors case) were shifted with respect to one another by half of the grating period in order to mimic the $2 \mathrm{D}$ photonic crystal with the optical axis directed along the diagonals of rhombi (see Fig. 2(b)) in case of the 1D modulation. In the $2 \mathrm{D}$ modulation case, care was taken to shift the periodic structure respectively in both transverse directions. In the unfolded structure the propagation of light succeeded along the diagonals of the cubic structure. The self-collimation in $3 \mathrm{D}$ is the most prominent along the diagonals of the cubic structure (as calculated in linear cases [24], shown experimentally [25], and predicted in the nonlinear case [26]). The resonator was illuminated by a $\mathrm{CW}$ laser beam (wavelength $532 \mathrm{~nm}$, beam width $2.5 \mathrm{~mm}$, power $15 \mathrm{~mW}$ ). A diffuser was placed in front of the front mirror of the resonator in order to generate a broad spatial spectrum of the illuminating radiation. Transmitted radiation was recorded by using the lens and a CCD or screen and photo camera. A lens of $58 \mathrm{~mm}$ focal distance was used to collect the transmitted radiation into a CCD camera for the far field recording.

\section{Mode structures of resonator with intracavity $\mathrm{PhC}$}

The transverse and longitudinal modes structure of homogeneously filled plane-mirror resonators (Fabry-Pérot resonators) is well known. Figure $4(\mathrm{a}, \mathrm{c})$ illustrates the resonator mode structure for the monochromatic radiation of frequency $\omega$ (and, correspondingly, of the modulus of the wavevector $|\mathbf{k}|=\omega / \mathrm{c}=2 \pi / \lambda)$. As the presence of the mirrors imposes the resonance conditions on the longitudinal component of the wavevector: $k_{\|}=2 \pi m /(2 l)$ ( $m$ is the longitudinal mode number, and $l$ is the linear cavity length), then the resonant transverse wavevector components become $k_{\perp}=\sqrt{|\mathbf{k}|^{2}-k_{\|}^{2}}=\sqrt{(\omega / c)^{2}-(\pi m / l)^{2}}$, resulting in a system of concentric Fresnel rings in the resonator angular (far field) transmission profile counted by the integer values of $m$. In homogeneous material the character of diffraction is fixed; therefore, there is not much freedom in varying the character of the
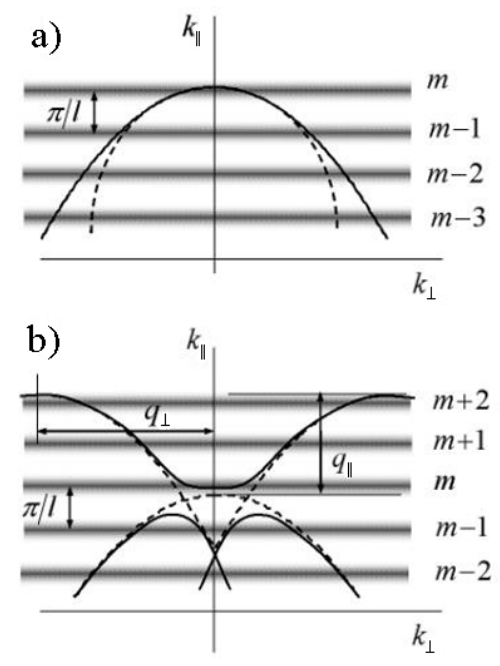

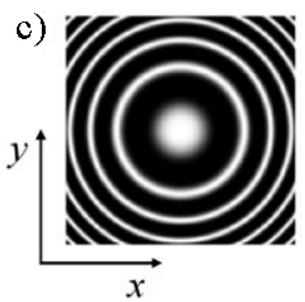

d)

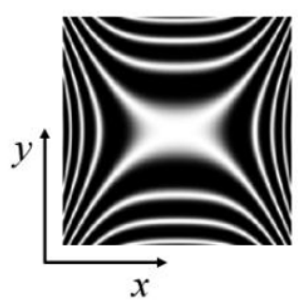

Fig. 4. Spatial dispersion curves (a) of monochromatic plane waves in homogeneous media and (b) of the Bloch modes in photonic crystals. The dispersion curve (b) is taken from [20], i. e. calculated by a standard technique of harmonic wave expansion. The dashed lines in (b) indicate the dispersion curves of uncoupled plane waves (or equivalently of the Bloch modes in the limit of vanishing index modulation $(s \rightarrow 0)$ ). The thick fuzzy lines in $(a, b)$ indicate the resonances of the resonator as characterized by the condition of the longitudinal component of the wavevector $k_{\|}=2 \pi \mathrm{m} /(2 \mathrm{l})$. (c) Fresnel ring structure of the homogeneously filled resonator. (d) Mode structure for the resonator with index modulation in one transverse direction. 
angular transmission profile. The mode structure (the system of Fresnel rings) is simply rescaled by varying the optical length of the resonator, i. e. the diffractivity of the resonator.

The idea behind the present article (as well as behind earlier works [20, 21]) is that the transverse and longitudinal mode structure of the plane-mirror resonator could be substantially modified filling the resonator with the material with a refraction index periodic in space, i. e. with a PhC. It is known that the character of diffraction can be substantially modified in the bulk propagation through the material with the periodic in space modulation of the refraction index of the media: the dominating orders of diffraction can change the sign or can convert to zero, resulting in the latter case in the effect of selfcollimation or subdiffraction [16, 17]. This allows us to expect that the mode structure of the resonator will also be respectively modified by the intracavity refraction index modulation. As the angular transmission profile of the resonator is closely related with its transverse mode structure, then the shape of the resonator angular transmission profile should also strongly depend on the character of the intracavity refraction index modulation. In particular, the angular transmission profile can be broadened or narrowed with respect to that of the "homogeneous" resonator, resulting in sub- and superdiffractive resonators. Below we perform a systematic numerical and experimental study of the angular transmission properties in such resonators.

Figure 4(b, d) illustrates the idea that the Fresnel ring structure could be substantially modified if the diffraction of the resonator is manipulated due to the periodic intracavity refraction index modulation. Here the two-dimensional intracavity $\mathrm{PhC}$ is considered, i. e. the index is considered to be modulated in one transverse and in one longitudinal direction (with respect to the optical axis of the resonator). In particular, the relatively broad angular areas of the resonances can be expected along the optical axis of the resonator when the plateau appears on the spatial dispersion curves (Fig. 4(b)) instead of the relatively narrow rings or a central spot (Fig. 4(a)). The 2D angular resonance profiles are more involved. Figure 4(d) illustrates the dispersion surface accounting for two transverse dimensions, where the refraction index is modulated in one transverse direction only (2D PhC). As the spatial dispersion surface resembles the saddle, then the resonances, being the hori- zontal cuts of the dispersion surface, result in the hyperbola-like angular transmission structure - in a strong contrast with the Fresnel ring structure of the homogeneously filled resonators in Fig. 4(c).

We also note that the light dynamics in the resonator modulated in a transverse direction only (no longitudinal index modulation) as studied previously in [27, 28] by applying the mean field models. Here in our study, the longitudinal modulation of the refraction index is considered (the longitudinal modulation index is necessary in order to obtain the subdiffractive, or self-collimating, regimes). The presence of the longitudinal modulation of the refraction index does not allow application of the mean field models like e. g. in [27, 28]; therefore, we developed a theoretical approach based on the multiple transfer matrix analysis. This analysis allows taking into account the multilongitudinal mode structure (multirings).

\section{Theoretical model}

For the theoretical-numerical analysis of the resonator modes we developed an approach based on the multiple scattering matrix technique. We analyse a roundtrip propagation of light along the resonator and calculate the transformation of the field on each of the elements consecutively: (i) diffraction on the modulated surface of the mirror, (ii) free space propagation between the mirrors (diffraction in homogeneous material), (iii) lateral shift of the periodic structure of the mirrors with respect to one another, and (iv) partial reflections from the mirrors. After calculating the field transformation in a resonator roundtrip (by applying the transformation operators, i. e. by multiplying by corresponding transformation matrices), we calculate the resonator transmission matrix by the standard techniques: by adding the entering plane wave to the resonators and by searching for a stationary state. This is essentially a classical approach to calculate the mode structure of the homogeneously filled Fabry-Pérot resonator, with the difference that the field transformations on each of the element in the resonator are now not scalars but operators (represented by matrices).

The periodic modulation of the mirror surface results in a set of diffraction components in the reflected light with transverse components of the wavevectors $\vec{k}_{\perp}+\vec{q}_{m, n}$, where $\vec{k}_{\perp}=\left(k_{x}, k_{y}\right)$ is the transverse wavevector of the incident light, and 
$\vec{q}_{m, n}=\left(m q_{x}, n q_{y}\right)$ are the multiples of the modulation wavevector. Strictly speaking, one should consider all the possible field harmonics; however, it comes out that consideration of the central component plus first-order sidebands is sufficient. Here, we consider one-dimensional modulation of the mirror surfaces (say in $x$ direction). The theory on the two-dimensional modulation case follows straightforwardly from the one-dimensional modulation case (can also be found in [21]). We approximate the resonating field in the following way:

$$
\begin{aligned}
& A(\mathbf{r})=e^{i k_{x} x}\left(a_{0}+a_{-1} e^{-i q_{x} x}+a_{+1} e^{i q_{x} x}\right. \\
& \left.+a_{-2} e^{-2 i q_{x} x}+a_{+2} e^{2 i q_{x} x}+\ldots\right)
\end{aligned}
$$

where $q_{x}$ is the wavevector of the index modulation in the transverse direction $x$. In case of two-dimensional modulation the wavevector of modulation $\vec{q}=\left(q_{x}, q_{y}\right)$ is to be considered. The plane-wave set (2) is tilted with respect to the optical axis, as represented by the factor in Eq.(1) with nonzero $k_{x}$. The field, for convenience, is further represented by the column vector of plane wave components:

$$
\vec{A}=\left(\ldots, a_{-2}, a_{-1}, a_{0}, a_{+1}, a_{+2}, \ldots\right)^{T} .
$$

Next, we list consecutively the field transformations in the resonator roundtrip.

Scattering by phase grating. The periodically modulated phase of the field on the reflection of the surface of the mirror couples the components of the field vectors. For the harmonic modulation the coupling occurs only between the neighbouring components. We introduce the phenomenological scattering coefficient $s_{x}$, which can be linked to microscopic parameters of the coating, such as the depth of the modulation, and the refraction index of the photoresist. However, it is more convenient to keep the macroscopic scattering parameter as it is directly linked with the experimentally accessible diffraction efficiency of the grating ( $s$ is the square root of the efficiency of scattering into the sidebands). The scattering matrix is:

$$
\hat{S}=\operatorname{Exp}\left(\begin{array}{ccccc}
0 & i s_{x} & 0 & 0 & 0 \\
i s_{x} & 0 & i s_{x} & 0 & 0 \\
0 & i s_{x} & 0 & i s_{x} & 0 \\
0 & 0 & i s_{x} & 0 & i s_{x} \\
0 & 0 & 0 & i s_{x} & 0
\end{array}\right) .
$$

Here and below we consider the five mode expansion for simplicity, which can be directly extended to the arbitrary number of components.

Free propagation. The free propagation over the linear length of the resonator is considered by the paraxial propagation equation:

$$
\partial_{z} A(\mathbf{r})=\frac{i}{2 k_{0}} \nabla_{\perp}^{2} A(\mathbf{r})
$$

where $\tilde{\mathrm{N}}_{\wedge}^{2}=\partial^{2} / \partial x^{2}+\partial^{2} / \partial y^{2}$ is the Laplace operator acting in the transverse plane. Substitution of expansion (1) into Eq. (4) yields the (uncoupled) equation system:

$$
\partial_{z} a_{m}=-\frac{i}{2 k_{0}}\left[\left(m q_{x}+k_{x}\right)^{2}+k_{y}^{2}\right] a_{m} .
$$

The integration of Eq. (5) over the linear resonator length results in the diagonal field transformation matrix:

$$
\begin{aligned}
& \vec{P}=e^{i l k_{0}} e^{-i L k_{y}^{2}} \cdot \operatorname{Diag}\left(\ldots, e^{-i L\left(k_{x}-2 q_{x}\right)^{2}}, e^{-i L\left(k_{x}-q_{x}\right)^{2}},\right. \\
& \left.e^{-i L k_{x}^{2}}, e^{-i L\left(k_{x}+q_{x}\right)^{2}}, e^{-i L\left(k_{x}+2 q_{x}\right)^{2}}, \ldots\right),
\end{aligned}
$$

where $L=l /\left(2 k_{0}\right)$ is the normalized length of the resonator representing its diffraction.

Lateral shift of the mirror. In the case when both mirrors are modulated and, importantly, when the second mirror is shifted in the lateral direction, we account for the lateral shift of the grating (determined by $m_{x}$ ) using the following trick. We fix the reference frame with the position of the first mirror. Then, for the calculation of the scattering from the laterally shifted second mirror, we change the reference frame by applying the operator

$$
\vec{M}=\operatorname{Diag}\left(\ldots, e^{-2 i m_{x} q_{x}}, e^{-i m_{x} q_{x}}, 1, e^{+i m_{x} q_{x}}, e^{+2 i m_{x} q_{x}}, \ldots\right) .
$$

Then, after calculating the scattering on the second mirror (by using Eqs. (3) or (4) in the new reference frame) we restore the original reference frame by applying $\vec{M}^{-1}$.

Resonator mirrors. Since the reflectivity is the same for all harmonic components of the wave, it is accounted in a standard way - by multiplication by a scalar $r$. Without losing generality in theory and in accordance with our experiment, we consider both mirrors of the same reflectivity. 
Resonator roundtrip. The variation of the field in a resonator roundtrip is calculated by applying consecutively all the operators discussed above:

$$
\hat{R}=r^{2} \hat{P} \hat{M}^{-1} \hat{S} \hat{M} \hat{P} \hat{S} .
$$

In special situations, when e. g. one mirror is not modulated, Eq. (8) respectively simplifies.

Resonator transmission function. The plane wave entering into the resonator is denoted in this vector form by $\vec{A}_{0}=(0,0,1,0,0)^{T}$ for the input field normalized to unity. Then, we calculate the radiation balance in one resonator roundtrip and analogously to the homogeneous Fabry-Pérot case we obtain

$$
\vec{A}=t(\hat{1}-\hat{R})^{-1} \overrightarrow{A_{0}}
$$

for the radiation at the entrance mirror. The only difference from the homogeneous Fabry-Perot case is that here we deal with the vectors of the wave components and transformation matrices, instead of complex scalar factors. Finally, the resonator transmission matrix is

$$
\hat{T}=t^{2}(\hat{1}-\hat{R})^{-1}=t^{2}\left(\hat{1}-r^{2} \hat{M}^{-1} \hat{P} \hat{S} \hat{M} \hat{P} \hat{S}\right)^{-1}
$$

The transmission for the homogeneous component is given by the element $T_{0,0}$ of the matrix (10). The scattering of the resonator into the sidebands harmonics is described by the corresponding offdiagonal column elements of the matrix: by $T_{-1,0}$ and $T_{+1,0}$ into the diffraction components in $x$ direction, and by $T_{0,-1}$ and $T_{0,+1}$ into the diffraction components in $y$ direction.

The transmission matrix (10) can be easily adopted for the single modulated mirror case by removing the operator of scattering from the modulated surface of the second mirror as well as the operator of the lateral shift:

$$
\hat{T}=t^{2}(\hat{1}-\hat{R})^{-1}=t^{2}\left(\hat{1}-r^{2} \hat{P} \hat{P} \hat{S}\right)^{-1} .
$$

The resonator transmission profiles calculated by (10), (11) give the basic properties expected: subdiffraction and superdiffraction, and are analysed in subsequent chapters.

\section{One-dimensional modulation of mirror surfaces}

The resonator configuration was built with the initial purpose to obtain the subdiffractive condition, i. e. the flattening of the dispersion curve as illustrated in Fig. 4. This occurs at around the resonance conditions for all the significant harmonic components of expansion (1). Although the final expression for the resonator transmission function (10) does not allow analytical interpretation of the results, the inspection of the free-propagation matrix (6) allows estimation of the parameters for this multiple resonance. The simultaneous resonance of at least three most relevant harmonic components is possible if different components belong to different longitudinal modes: the central mode $a_{0}$ belongs to a particular longitudinal mode $n$ and the sideband modes belong to the longitudinal mode with the index $n-1$. From (6) it follows that the conditions must hold: $l k_{0} \approx \pi$ n (the resonance condition for the central component) and $L q_{x}^{2} \approx \pi$ (the resonance condition for the sidebands). The latter means $l q_{x}^{2} / k_{0} \approx 2 \pi$, or equivalently $l \lambda / d^{2}{ }_{x, y} \approx 1$, where $d_{x}=2 \pi / q_{x}$ are the corresponding spatial periods of the modulation of the surfaces of the mirrors, and $l$ (we repeat) is the linear length of the resonator.

The latter conditions were indicators for selecting the resonator length in our experiments. The "rough tuning" of the resonator length around the critical one $l_{\mathrm{cr}}=d_{x}{ }^{2} / \lambda$ ensures the multiple resonance condition, i. e. all field harmonics are simultaneously in or out of the resonance, depending on the fine tuning of the resonator. The other condition $l k_{0} \approx \pi n$ was considered for the fine (submicron) tuning of the resonator length in order to simultaneously tune all harmonics to the resonance. The fine tuning is characterized by the roundtrip phase of the resonator: $\varphi=2 l k_{0}-2 \pi n$.

\subsection{Single modulated mirror}

We start our analysis from the case when only one resonator mirror has a modulated surface. Figure 5 shows the transmission of the resonator as calculated according to (11). The figure shows that depending on the parameters of the resonator (essentially, resonator length and mirror reflectivity) two relevant cases are obtained: subdiffractive (Fig. 5(a)) and superdiffractive (Fig. 5(c)). In the subdiffractive case, transmission shows distribution broader than in the reference case of the homogeneous resonator. In the superdiffractive case, the transmission profile is narrower. As the modulation of mirrors is one-dimensional and is directed horizontally ( $\mathrm{x}$ direction), 


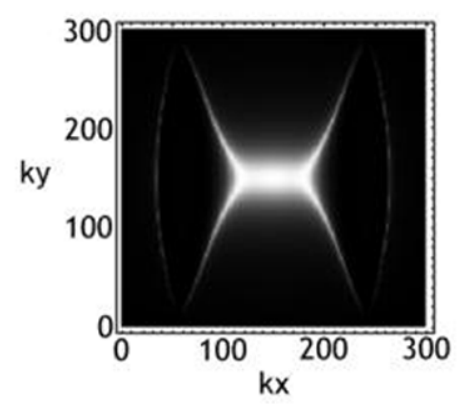

a)
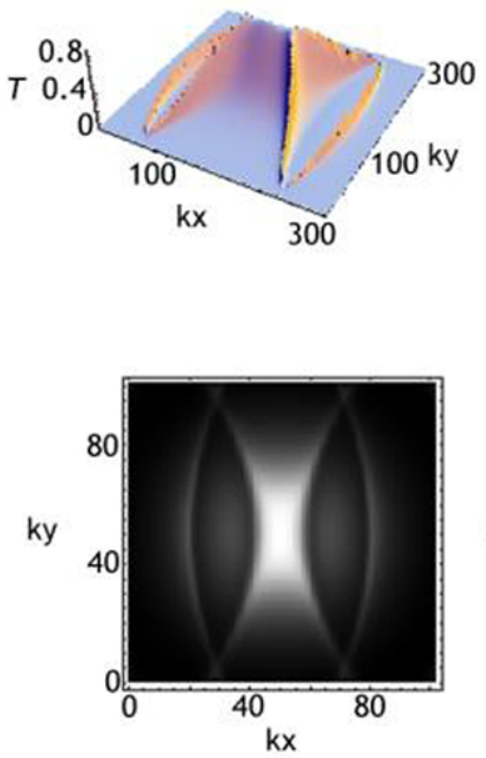

c)

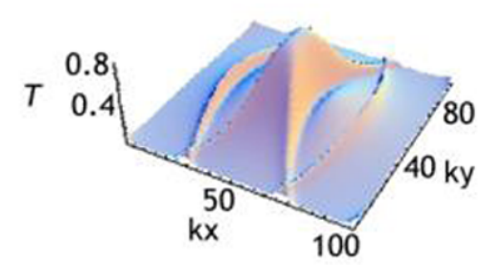

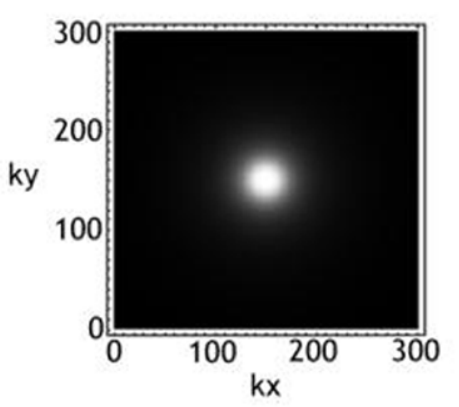

b)
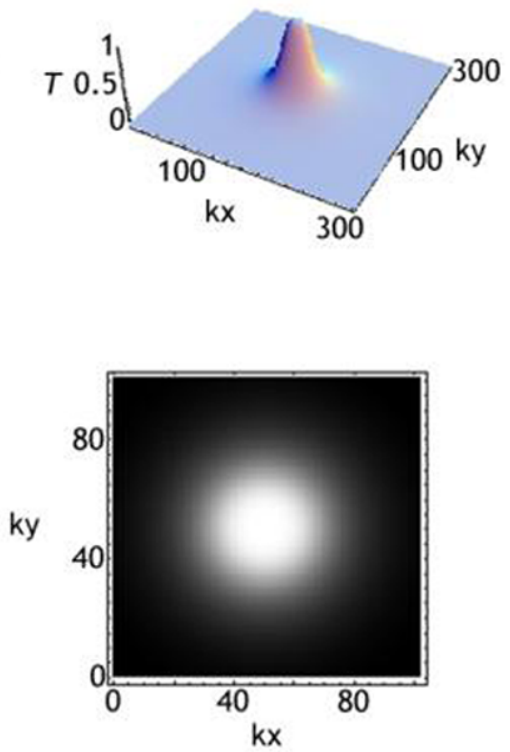

d)

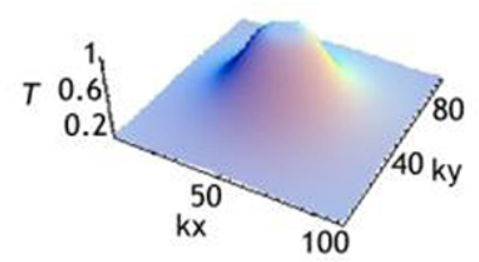

Fig. 5. Transmission profile of a $\mathrm{PhC}$ resonator with a single modulated mirror as calculated numerically from (11): (a) subdiffraction angular transmission profiles at normalized resonator length $d=4.2$ with parameters: $s=0.4, t=0.015$, $\varphi=-0.1$, (b) transmission profile in corresponding homogeneous case at $s=0$, $\varphi=0$, (c) superdiffraction angular transmission profiles at length $d=3.7$ with parameters: $s=0.4, t=0.2, \varphi=-0.25$, and (d) corresponding homogeneous case of (c). then the transformation of the transmission profile occurs only in this direction.

Below we analyse these two limiting cases in detail, by calculating and plotting the spatial characteristics of the transmission profile on a crosssection $k_{Y}=0$. The resonator transmission profile depends on cavity length $d$ and fine tuning, or phase $\varphi$, diffraction efficiency $s$, mirror transmission $t$. Figure $6(a, c, e)$ represents superdiffraction, Fig. 6 (b, d, f) represents subdiffraction. The black line in Fig. 6 indicates transmission of a homogeneous cavity.

At a low diffraction efficiency (Fig. 6(a)) $s=0.1$ and for $3.3 \leq d \leq 3.8$ the top value of transmission (for on-axis radiation) exceeds 0.9 , but the peripheral parts of the transmission profile are relatively strong. If the cavity length is smaller, the top transmission decreases rapidly. For the longer resonators, the angular transmission spectrum is broader than a homogeneous resonator. Also, peripheral parts decrease and the transmission profile becomes smoother (see Fig. 6(c)). For diffraction efficiency $s=0.35$ (Fig. 6(e)) peripheral parts become irrelevant; however, the top values of transmission decrease down to 0.8 .

The subdiffractive regime is obtained for the range of cavity length $4.5 \leq d \leq 5$. If diffraction efficiency is low $(s=0.1)$ subdifraction does not occur (Fig. 6(b)) and the top transmission is close to 1 . Higher diffraction efficiency makes transmission broader than the homogeneous one (Fig. 6(d)). The dispersion curve 

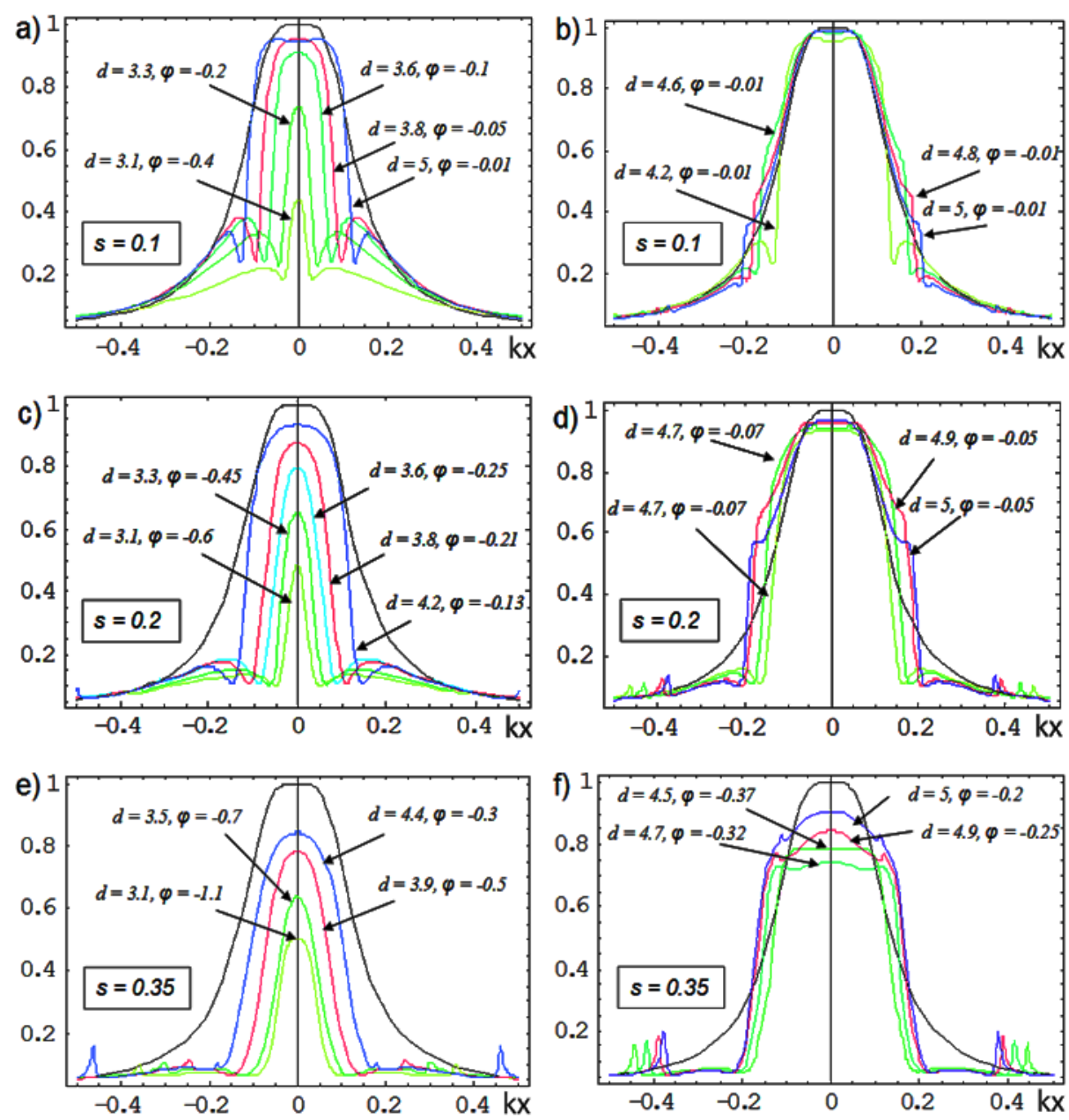

Fig. 6. Resonator transmission cross-section at $k_{y}=0$ for different cavity parameters: (a), (c), (e) represent superdiffraction case, (b), (d), (f) represent subdiffraction. Resonator transmission $t=0.1$ is fixed for all cases. Resonator phase is tuned for maximum transmission.

has abrupt edges so the cavity still can be used as a spatial filter. In case of high diffraction efficiency (Fig. 6(f)) dispersion exhibits the quadratic-like shape with a nearly flat plateau. It could be noted that

in case of a high diffraction efficiency the shape of the dispersion curve is very sensitive to cavity phase.

The influence of the cavity finesse on the angular transmission profile is summarized in Fig. 7. Two
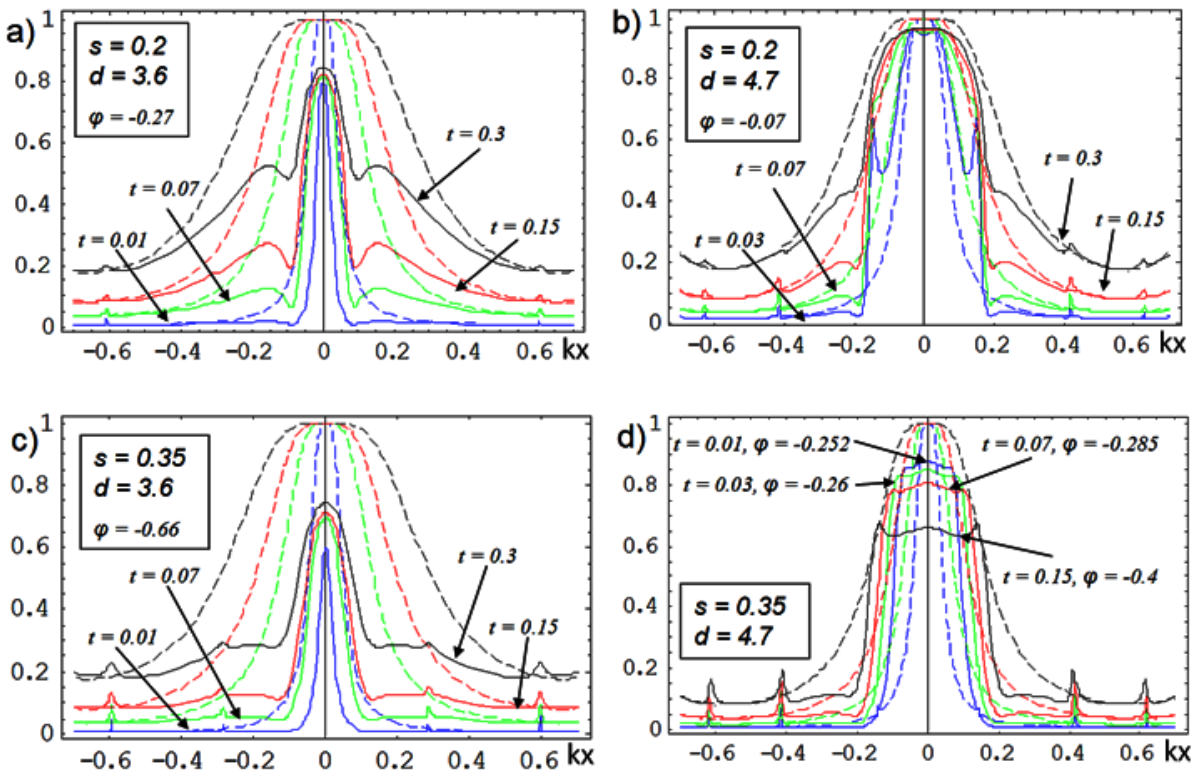

Fig. 7. Resonator transmission cross-section at $k_{y}=0$ depending on cavity mirror transmission $t$ : (a), (c) represent superdiffraction case, and (b), (d) represent subdiffraction. Transmission of homogeneously filled resonator is indicated by dashed lines. 
sets of parameters are used to explore the angular filtering and subdiffraction. The transmission of a homogeneously filled resonator is indicated by dashed lines. Higher cavity finesse (lower mirror transmission) reduces borders around the central peak in all cases. Figure $7(a, c)$ shows the spatial filtering regime indicating that filtering is possible at any cavity mirror transmission or grating diffraction efficiency. Lower mirror transmission reduces the peripheral part of the transmission function but does not affect central peak intensity very much. After filtering, on-axis intensity decreases by the factor of $20-40 \%$.

Contrarily to filtering, the subdiffraction regime cannot be realized in the cavity with high transmission mirrors (Fig. 7(b, d)). Cavity transmission for diffraction efficiency $s=0.2$ and constant phase is shown in Fig. 7(b). Half intensity width is almost unchanged, but for low transmission mirrors three intensity peaks appear. In Fig. 7(d) the phase is attenuated to form a nearly flat top. It is evident from this picture that the subdiffraction regime takes place in a cavity with a relatively high finesse and diffraction efficiency.
In $1 \mathrm{D}$ experiments we used two different geometries: double modulated mirror resonator (Fig. 2(a)) and single modulated mirror resonator (Fig. 2(b)). Next, we analyse the resonator transmission function obtained at around the critical length of the resonator and by varying the fine tuning condition as characterized by the roundtrip phase $\varphi=2 l k_{0}-2 \pi n$. The roundtrip phase in experiments could not be directly determined and was indirectly restored from the comparison with the corresponding numerical plots (with the explicitly defined phase).

For single modulated mirror cavity experiments a 1D modulated $4 \mu \mathrm{m}$ period mirror was used which implies the critical cavity length of $l_{\mathrm{cr}}=d^{2} / \lambda=30 \mu \mathrm{m}$. The modulation profile is shown in Fig. 3(b). Diffraction efficiency of grating was $27 \%$ in zero order, $30.5 \%$ in \pm first order, and $5 \%$ in the \pm second order maxima. Experimental results are in very good correspondence with numerical results (Fig. 8). In Fig. 8(b), the filtering (superdiffractive) regime is evident. All experimentally obtained transmission profiles correspond to the cases of high resonator mirror transmission (i. e. low resonator finesse). As it comes out from resonator parameter analysis a)
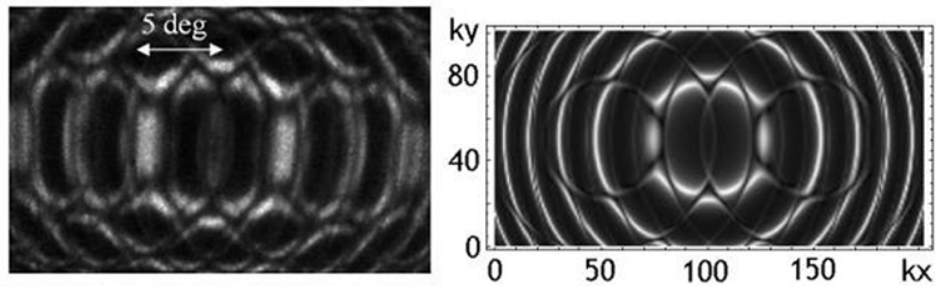

b)

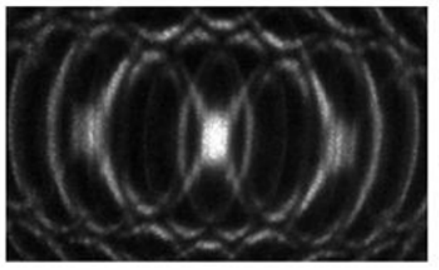

c)

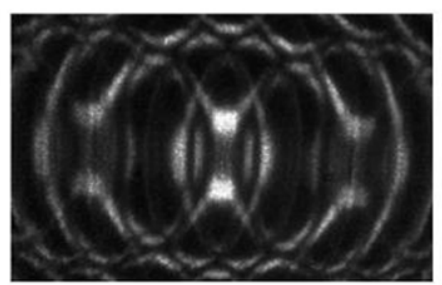

d)

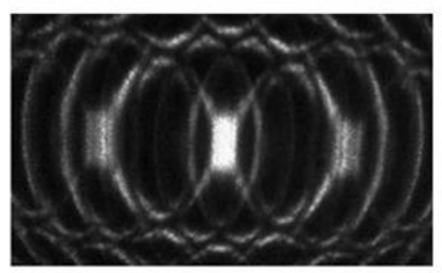

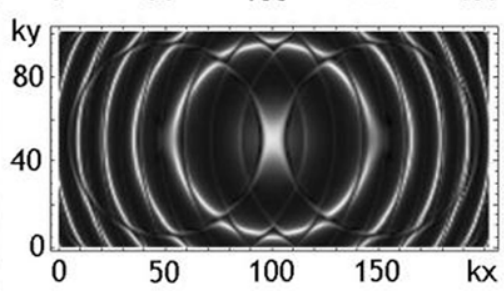
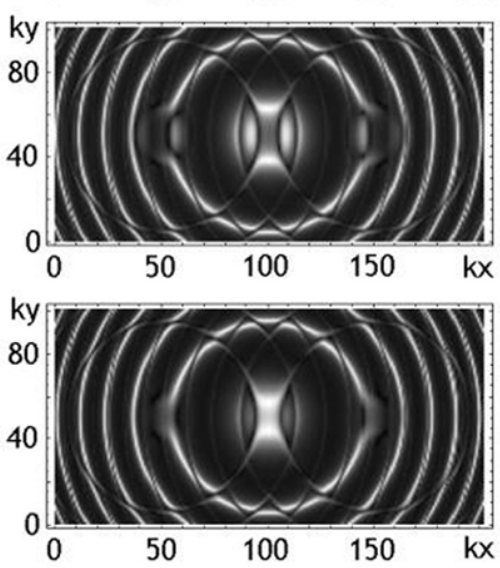

$\varphi=0.7 \pi$

$\varphi=1.9 \pi$

$\varphi=0.1 \pi$

$\varphi=2 \pi$
Fig. 8. Far field of radiation transmitted through the resonator with a $1 \mathrm{D}$ single modulated mirror as recorded experimentally and calculated numerically. Parameters: $s=0.4, t=0.2$, physical and theoretical resonator length $l=35 \mu \mathrm{m}(\mathrm{a}, \mathrm{b})$ and $l=38 \mu \mathrm{m}(\mathrm{c}, \mathrm{d})$. 
(see discussion above and Figs. 6, 7) the subdiffraction regime cannot be realized if the cavity finesse is low (mirror transmission is large, $t>0.2$ ). Although mirror transmission is relatively small, $t=0.015$, the scattering losses from the modulated surface lower the net finesse of the resonator. The modulation profile of the grating is not perfectly harmonic (Fig. 3(b)) and diffraction efficiency to higher order maxima is $10 \%$. This causes losses and reduces the net finesse of a cavity.

Here we also note the advantages of a single modulated mirror cavity. From an experimental point of view a cavity with a single modulated mirror is attractive because of simplicity of the resonator. It does not need gratings to be shifted by half of a period and does not need to keep mirror grating precisely parallel. On the other hand, a mirror with no grating can be covered with a nonlinear or active material.

\section{2. Double modulated mirror}

The double modulated mirror case was already reported in [21]. For comparison with the single modulated mirror case we present here the resonator transmission profiles, both calculated by (10) and recorded experimentally. The modulation period of a double mirror resonator mirror surface was $d_{x}=2 \mu \mathrm{m}$ (modulation profile is shown in Fig. 3(b)), which implies the critical cavity length $l_{c r}=d^{2} / \lambda=7.5 \mu \mathrm{m}$. The diffraction efficiency of a grating was $52 \%$ in zero order, $16 \%$ in +-first order, and $4 \%$ in the \pm second order maxima. Again, a good

a)

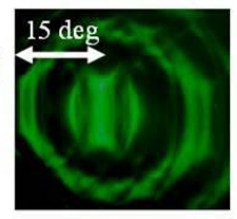

b)
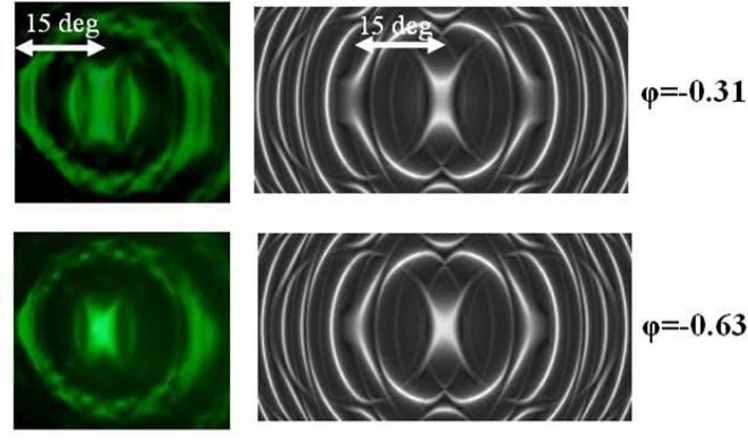

Fig. 9. Far field of radiation transmitted through the resonator with 1D modulated mirror surface as recorded experimentally and calculated numerically from (10). The lateral shift of the mirrors is half of the modulation period. Parameters used are: $s=0.4, t=0.2, d=4.0$, $m=\pi$. The resonator roundtrip phase was varied to fit the experimental plots. qualitative correspondence between results recorded experimentally (left column in Fig. 9) and calculated numerically (right column in Fig. 9) is envisaged. The case in Fig. 9(b) corresponds to the multiple resonances of all three waves, i. e. to the case when the frequency of the plateau of the dispersion curve is fine-tuned to the resonance. The case in Fig. 9(a) is characteristic of situations when the resonator is fine-tuned from the resonance.

\section{2D modulation of mirror surfaces}

In $2 \mathrm{D}$ experiments the period of modulation of mirror surface was $d_{x}=4 \mu \mathrm{m}, \mathrm{d}_{\mathrm{y}}=4 \mu \mathrm{m}$ (quadratic structure), which implies the critical cavity length of $l_{\mathrm{cr}}=d^{2} / \lambda=30 \mu \mathrm{m}$. Figure 10 shows the resonator transmission function obtained at around the critical length of the resonator and by varying the fine tuning condition.

Also, in a 2D modulation case a good qualitative correspondence with numerically obtained results was observed. Here again, the resonant case was obtained, characterized by a relatively a)

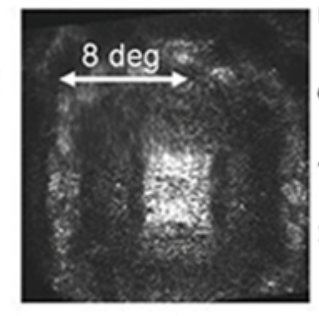

b)

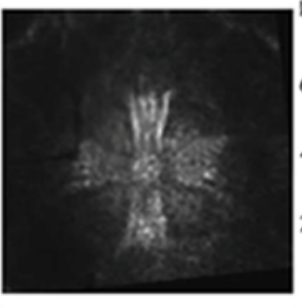

c)

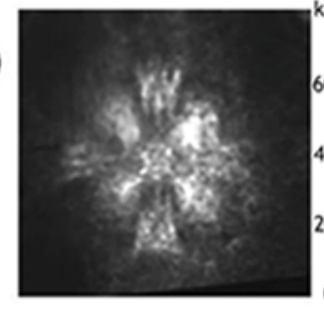

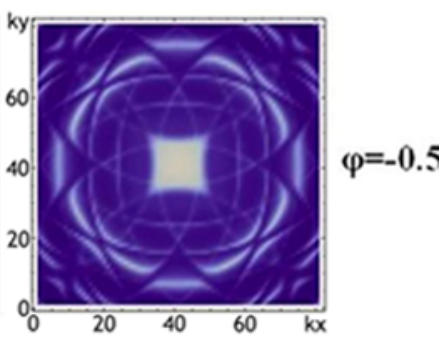
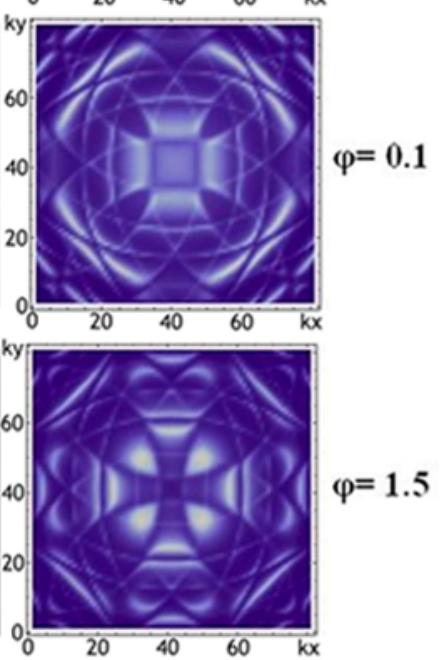

Fig. 10. Far field of radiation transmitted through the resonator with $2 \mathrm{D}$ modulated mirrors as recorded experimentally and calculated numerically (10). The lateral shift of the mirrors is half of the modulation period in both directions $\left(m_{x}=m_{y}=\pi\right)$. Parameters: $s=0.4 ; t=0.2 ; d=4.5$. 
flat (homogeneous) and broad resonator transmission function. This case is analogous to the case in Fig. 8(b), however here obtained as a simultaneous resonance of five waves. Also, in analogy to a $1 \mathrm{D}$ case the typical off-resonance transmission distributions were obtained as shown in Fig. 10(b, c).

The multiple resonance condition and the appearance of plateau can be also interpreted in a geometric way. Differently from 1D case, here it seems that the most homogeneous plateau appears under participation of the two resonant rings from the diffraction maxima, as indicated in Fig. 11. This means that also the longitudinal $n-2$ modes participate in formation of subdiffractive pattern.

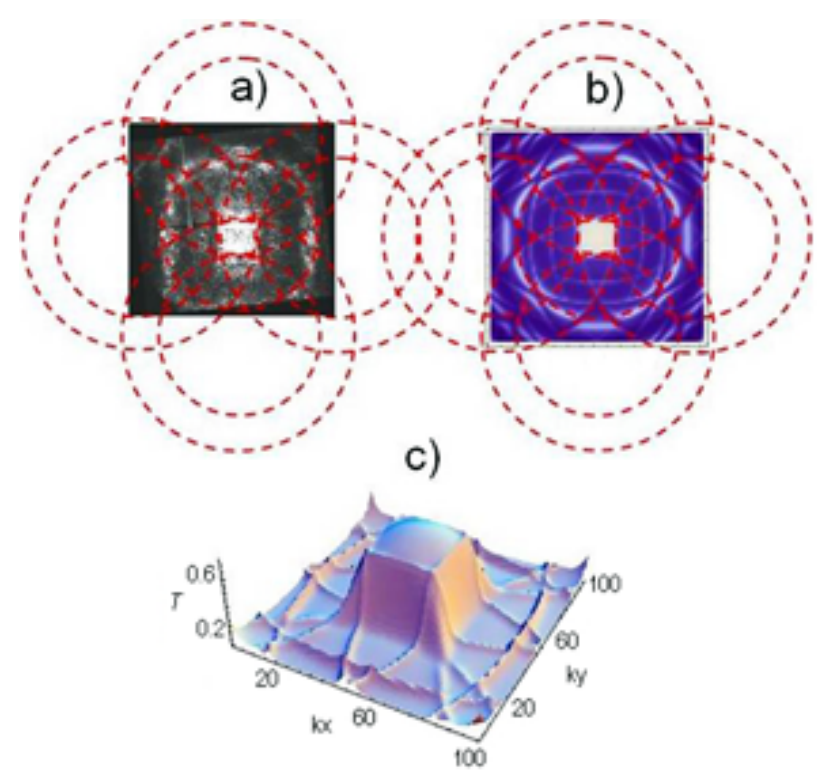

Fig. 11. Ring structure of 2D modulated mirror cavity. Experimental results (a) and numerical simulation (b) are as taken from Fig. 10(a). Dashed red rings indicate imaginary place of transmission rings around diffraction maxima. (c) 3D resonator transmission structure showing the role of two resonance rings, as obtained from numerics of (10).

The resonator transmission pattern was sensible with respect to the lateral shift of the resonator mirrors with respect to one another. Figure 12 reports the result of the study of the lateral shift. As expected, the lateral shift by the value different than $m_{x, y}=\pi, 0.5 \pi$ results in an asymmetric pattern with respect to the direction with broken symmetry (Fig. 12(a)). The lateral shifts of $m_{x, y}=0$ and $m_{y}=\pi$ result in different patterns, as shown in Fig. 12 (b). a)
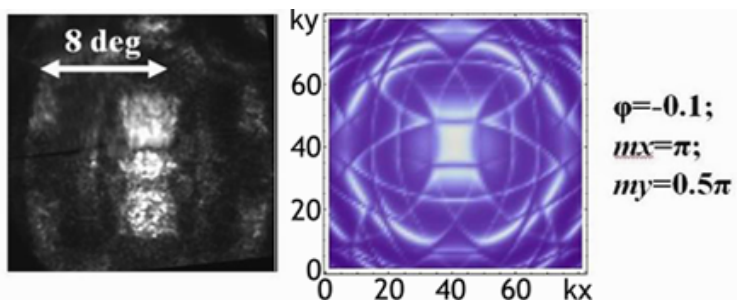

b)
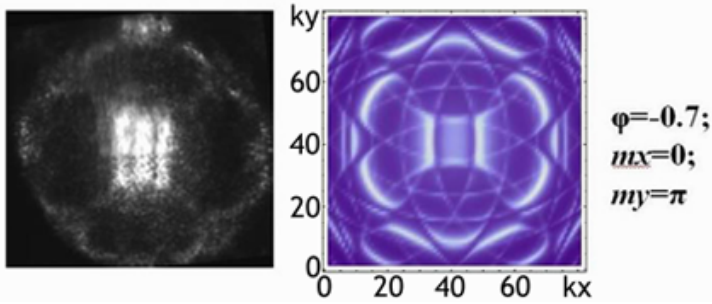

Fig. 12. Far field of radiation transmitted through the resonator with $2 \mathrm{D}$ modulated mirrors and different lateral shift as recorded experimentally and calculated numerically (10). Parameters: $s=0.4 ; t=0.2 ; d=4.5$.

\section{Conclusions}

To conclude with, we built a $\mathrm{PhC}$ resonator with the intracavity modulation of the refraction index, i. e. resonator containing one longitudinal period of a PhC. We developed the method of calculation of such resonator based on the scattering matrix theory and reproduced the experimentally observed transmission patterns by numerical integration of the developed model. We demonstrated experimentally the basic properties expected, i. e. hyperbolic shape transmission patterns in case of $1 \mathrm{D}$ modulation of mirror surfaces and square shape patterns in case of 2D modulation of mirror surfaces.

The $\mathrm{PhC}$ resonator shows the basic properties expected - the relatively flat angular transmission profile. The maximum transmission area is of quadratic shape due to a quadratic symmetry of the modulation of mirror surfaces. More symmetric modulation patterns (hexagonal or octagonal) are expected to result in a more isotropic transmission spot.

We also analysed the resonator mirror transmission, grating scattering, and resonator length influence on cavity transmission properties. We pointed out that high reflection $(R>95 \%)$ mirrors (i. e. high finesse cavity) are necessary for subdiffraction resonator properties.

\section{Acknowledgements}

We acknowledge cooperation with M. Anrulevičius, A. Guobiene, and T. Tamulevičius. The work was 
financially supported by Spanish Ministerio de Educación y Ciencia and European FEDER (project FIS2011-29734-C02-01), Lithuanian State Science and Studies Foundation through Project No. T-08208, and European Union Structural Funds project "Postdoctoral Fellowship Implementation in Lithuania".

\section{Appendix}

Herein we give a short historical survey on transverse pattern formation in lasers and nonlinear optical resonators.

The first hints on spontaneous pattern formation in broad aperture lasers and nonlinear resonators appeared in the early 1970s when the relation between the coherent laser field and fluids/superfluids was recognized [29]. Also, filamentation of light in focusing media and, in more sophisticated cases, formation of bright spatial solitons [30] can be attributed to the initial works on nonlinear light patterns. However, really detailed studies of nonlinear patterns started in the late 1980s and early 1990s. In [31] nontrivial stationary and dynamical transverse mode formations in laser beams were shown. In [32] it was recognized that MaxwellBloch equations for a laser admit vortex solutions, which soon were confirmed experimentally [33]. These pioneering works were followed by an increasing number of investigations, mostly attempts to understand nonlinear pattern formation by deriving the order parameter equation for a laser and other broad aperture nonlinear resonators. The Ginzburg-Landau equation as an order parameter equation for a laser was already derived in [29] in a very simple approximation. More sophisticated order parameter equations were derived, such as modified Ginzburg-Landau equation [34], SwiftHohenberg equation [35, 36]. The order parameter equations were also derived for other types of nonlinear resonators: resonator with a nonlinear photorefractive crystal [37], resonator with parametric nonlinearity [38, 39], and passive driven nonlinearKerr resonator [40, 41].

In parallel to the patterns in nonlinear resonators, the nonlinear patterns in different optical configurations were studied, such as mirrorless configuration with unidirectional light propagation in nonlinear media, leading to filamentation effects and formation of bright solitons [42], optical vortices [43], counterpropagating configuration leading to hexagons [44], systems with a feedback loop, showing a large variety of extended patterns [45, 46.

Coming back to nonlinear resonators, various phenomena have been studied. One exotic formation is an optical vortex [32, 33, 47-50] which can show chaotic dynamics (vortex mediated turbulence) [51, 52], the vortices that can arrange in regular vortex lattices [53-55], and the like. Another relevant localized structure is a bright dissipative soliton in bistable nonlinear resonators, in bistable lasers (with saturable absorbers) [56-58], in passive nonlinear resonators [59], in optical parametric oscillators [60, 61], and in vertical surface cavity emission lasers [62-64]. Also, phase solitons (related with the bistability of the phase) should be mentioned as interesting localized formations of light [65-67]. Very different issues have been considered, such as threedimensional patterns (localized bullets) [68, 69, "rocked" resonators [70,71], to mention a few.

Generally, the character of the above-discussed light patterns in nonlinear resonators is strongly dependent on diffraction of resonators. Some early attempts were made to eliminate the diffraction of the resonator by using self-imaging geometries [72]. As an alternative, the modulation of the intracavity refraction index was proposed to manipulate diffraction, bringing to subdiffractive solitons [73, 74], hyperbolic patterns [75, 76, or elimination of spatial instabilities of the patterns [27, 28]. Further works on the topic of modulated resonators (or resonators filled with $\mathrm{PhCs}$ ) are reviewed in the introductory part of the main part of the article.

\section{References}

[1] V.J. Sanchez-Morcillo and K. Staliunas, Transverse Patterns in Nonlinear Optical Resonators (Springer, 2003).

[2] G. Nicolis and I. Prigogine, Self-organization in Nonequilibrium Systems: From Dissipative Structures to Order through Fluctuations (Wiley, New York, 1977).

[3] M.C. Cross and P.C. Hohenberg, Pattern formation outside of equilibrium, Rev. Mod. Phys. 65(3), 851-1112 (1993).

[4] K. Staliunas, S. Longhi, and G.J. de Valcárcel, Faraday patterns in Bose-Einstein condensates, Phys. Rev. Lett. 89(21), 210406 (2002).

[5] H. Saito and M. Ueda, Dynamically stabilized bright solitons in a two-dimensional Bose-Einstein condensate, Phys. Rev. Lett. 90(4), 040403 (2003). 
[6] F.K. Abdullaev, J.G. Caputo, R.A. Kraenkel, and B.A. Malomed, Controlling collapse in BoseEinstein condensates by temporal modulation of the scattering length, Phys. Rev. A 67(1), 013605 (2003).

[7] F.K. Abdullaev, A.M. Kamchatnov, V.V. Konotop, and V.A. Brazhnyi, Adiabatic dynamics of periodic waves in Bose-Einstein condensates with time dependent atomic scattering length, Phys. Rev. Lett. 90(23), 230402 (2003).

[8] D.E. Pelinovsky, P.G. Kevrekidis, and D.J. Frantzeskakis, Averaging for solitons with nonlinearity management, Phys. Rev. Lett. 91(24), 240201 (2003).

[9] A.R. Davoyan, I.V. Shadrivov, and Y.S. Kivshar, Self-focusing and spatial plasmon-polariton solitons, Opt. Express 17(24), 21732-21737 (2009).

[10]B. Stein, J.-Y. Laluet, E. Devaux, C. Genet, and T.W. Ebbesen, Surface plasmon mode steering and negative refraction, Phys. Rev. Lett. 105(26), 266804 (2010).

[11]B. Stein, E. Devaux, C. Genet, and T.W. Ebbesen, Self-collimation of surface plasmon beams, Opt. Lett. 37(11), 1916-1918 (2012).

[12] K.Y. Bliokh, Y.P. Bliokh, V. Freilikher, S. Savel'ev, and F. Nori, Colloquium: unusual resonators: plasmonics, metamaterials, and random media, Rev. Mod. Phys. 80(4), 1201-1213 (2008).

[13]R. Iliew, C. Etrich, T. Pertsch, F. Lederer, and K. Staliunas, Subdiffractive all-photonic crystal Fabry-Perot resonators, Opt. Lett. 33(22), 26952697 (2008).

[14]K. Staliunas, O. Egorov, Y.S. Kivshar, and F. Lederer, Bloch cavity solitons in nonlinear resonators with intracavity photonic crystals, Phys. Rev. Lett. 101(15), 153903 (2008).

[15]C. Etrich, R. Iliew, K. Staliunas, F. Lederer, and O.A. Egorov, $A b$ initio dissipative solitons in an all-photonic crystal resonator, Phys. Rev. A 84(2), 021808.

[16]R. Zengerle, Light propagation in singly and doubly periodic planar waveguides, J. Mod. Opt. 34(12), 1589-1617 (1987).

[17]H. Kosaka, T. Kawashima, A. Tomita, M. Notomi, T. Tamamura, T. Sato, and S. Kawakami, Selfcollimating phenomena in photonic crystals, Appl. Phys. Lett. 74(9), 1212-1214 (1999).

[18]K. Staliunas and V.J. Sánchez-Morcillo, Spatial filtering of light by chirped photonic crystals, Phys. Rev. A 79(5), 053807 (2009).

[19] L. Maigyte, T. Gertus, M. Peckus, J. Trull, C. Cojocaru, V. Sirutkaitis, and K. Staliunas, Signatures of light-beam spatial filtering in a threedimensional photonic crystal, Phys. Rev. A 82(4), 043819 (2010).

[20]K. Staliunas, M. Peckus, and V. Sirutkaitis, Sub- and superdiffractive resonators with intracavity photonic crystals, Phys. Rev. A 76, 051803(R) (2007).
[21]M. Peckus, R. Rogalskis, M. Andrulevicius, T. Tamulevicius, A. Guobiene, V. Jarutis, V. Sirutkaitis, and K. Staliunas, Resonators with manipulated diffraction due to two- and three-dimensional intracavity photonic crystals, Phys. Rev. A 79(3), 033806 (2009).

[22] K. Staliunas and R. Herrero, Nondiffractive propagation of light in photonic crystals, Phys. Rev. E 73, 016601 (2006).

[23] Y. Loiko, C. Serrat, R. Herrero, and K. Staliunas, Quantitative analysis of subdiffractive light propagation in photonic crystals, Opt. Commun. 269(1), 128-136 (2007).

[24] R. Iliew, C. Etrich, and F. Lederer, Self-collimation of light in three-dimensional photonic crystals, Opt. Express 13(18), 7076-7085 (2005).

[25]Z. Lu, S. Shi, J.A. Murakowski, G.J. Schneider, C.A. Schuetz, and D.W. Prather, Experimental demonstration of self-collimation inside a threedimensional photonic crystal, Phys. Rev. Lett. 96(17), 173902-4 (2006).

[26] K. Staliunas, R. Herrero, and G.J. de Valcárcel, Arresting soliton collapse in two-dimensional nonlinear Schrödinger systems via spatiotemporal modulation of the external potential, Phys. Rev. A 75(1), 011604 (2007).

[27] D. Gomila, R. Zambrini, and G.-L. Oppo, Photonic band-gap inhibition of modulational istabilities, Phys. Rev. Lett. 92(25), 253904 (2004).

[28]D. Gomila and G.-L. Oppo, Coupled-mode theory for photonic band-gap inhibition of spatial instabilities, Phys. Rev. E 72(1), 016614-8 (2005).

[29] R. Graham and H. Haken, Laserlight - first example of a second-order phase transition far away from thermal equilibrium, Zeit. für Physik 237, 31-46 (1970).

[30] S.A. Akhmanov, R.V. Khoklov, and A.P. Sukhorukov, in: Laser Handbook, eds. F.T. Arechi and E.O. Schultz-DuBois, vol. 2 (North-Holland, Amsterdam, 1972).

[31] L.A. Lugiato, C. Oldano, and L.M. Narducci, Cooperative frequency locking and stationary spatial structures in lasers, J. Opt. Soc. Am. B 5(5), 879-888 (1988).

[32] P. Coullet, L. Gil, and F. Rocca, Optical vortices, Opt. Commun. 73(5), 403-408 (1989).

[33] M. Brambilla, F. Battipede, L.A. Lugiato, V. Penna, F. Prati, C. Tamm, and C.O. Weiss, Transverse laser patterns. I. Phase singularity crystals, Phys. Rev. A 43(9), 5090-5113 (1991).

[34] G.-L. Oppo, G. D’Alessandro, and W.J. Firth, Spatiotemporal instabilities of lasers in models reduced via center manifold techniques, Phys. Rev. A 44(7), 4712-4720 (1991).

[35]K. Staliunas, Laser Ginzburg-Landau equation and laser hydrodynamics, Phys. Rev. A 48(2), 1573 (1993). 
[36] P.K. Jakobsen, J.V. Moloney, A.C. Newell, and R. Indik, Space-time dynamics of wide-gain-section lasers, Phys. Rev. A 45(11), 8129 (1992).

[37] K. Staliunas, M.F.H. Tarroja, G. Slekys, C.O. Weiss, and L. Dambly, Analogy between photorefractive oscillators and class-A lasers, Phys. Rev. A 51(5), 4140 (1995).

[38] S. Longhi and A. Geraci, Swift-Hohenberg equation for optical parametric oscillators, Phys. Rev. A 54(5), 4581 (1996).

[39] G.J. de Valcarcel, K. Staliunas, E. Roldan, and V.J. Sanchez-Morcillo, Transverse patterns in degenerate optical parametric oscillation and degenerate four-wave mixing, Phys. Rev. A 54(2), 1609 (1996).

[40] P. Mandel, M. Georgiou, and T. Erneux, Transverse effects in coherently driven nonlinear cavities, Phys. Rev. A 47(5), 4277-4286 (1993).

[41] M. Tlidi, M. Georgiou, and P. Mandel, Transverse patterns in nascent optical bistability, Phys. Rev. A 48(6), 4605-4609 (1993).

[42] M. Haelterman and A.P. Sheppard, Polarization instability, multistability and transverse localized structures in Kerr media, Chaos Soliton Fract. 4(8-9), 1731-1743 (1994).

[43] G.A. Swartzlander, Jr. and C.T. Law, Optical vortex solitons observed in Kerr nonlinear media, Phys. Rev. Lett. 69(17), 2503-2506 (1992).

[44] W.J. Firth and C. Paré, Transverse modulational instabilities for counterpropagating beams in Kerr media, Opt. Lett. 13(12), 1096-1098 (1988).

[45]S.A. Akhmanov, M.A. Vorontsov, V.Y. Ivanov, A.V. Larichev, and N.I. Zheleznykh, Controlling transverse-wave interactions in nonlinear optics: generation and interaction of spatiotemporal structures, J. Opt. Soc. Am. B 9(1), 78-90 (1992).

[46] N.I. Zheleznykh, M. Le Berre, E. Ressayre, and A. Tallet, Rotating spiral waves in a nonlinear optical system with spatial interactions, Chaos Soliton Fract. 4(8-9), 1717-1728 (1994).

[47] K. Staliunas, Dynamics of optical vortices in a laser beam, Opt. Commun. 90(1-3), 123-127 (1992).

[48]D. Rozas, C.T. Law, and J.G.A. Swartzlander, Propagation dynamics of optical vortices, J. Opt. Soc. Am. B 14(11), 3054-3065 (1997).

[49] Yu. S. Kivshar and E.A. Ostrovskaya, Optical vortices: Folding and twisting waves of light, Opt. Photon. News 12, 24-28 (2001).

[50]K. Staliunas, Vortices and dark solitons in the twodimensional nonlinear Schrödinger equation, Chaos Soliton Fract. 4(8-9), 1783-1796 (1994).

[51]F.T. Arecchi, G. Giacomelli, P.L. Ramazza, and S. Residori, Vortices and defect statistics in twodimensional optical chaos, Phys. Rev. Lett. 67(27), 3749-3752 (1991).

[52] L. Gil, K. Emilsson, and G.L. Oppo, Dynamics of spiral waves in a spatially inhomogeneous Hopf bifurcation, Phys. Rev. A 45(2), R567-R570 (1992).
[53]K. Staliunas and C.O. Weiss, Tilted and standing waves and vortex lattices in class-A lasers, Physica D: Nonlinear Phenom. 81(1-2), 79-93 (1995).

[54]D. Hennequin, L. Dambly, D. Dangoisse, and P. Glorieux, Basic transverse dynamics of a photorefractive oscillator, J. Opt. Soc. Am. B 11(4), 676-684 (1994).

[55] K. Staliunas and C.O. Weiss, Nonstationary vortex lattices in large-aperture class B lasers, J. Opt. Soc. Am. B 12(6), 1142-1149 (1995).

[56]S. Fauve and O. Thual, Solitary waves generated by subcritical instabilities in dissipative systems, Phys. Rev. Lett. 64(3), 282-284 (1990).

[57] N.N. Rosanov, Progress in Optics (North-Holland, Amsterdam, 1996).

[58] G. Slekys, K. Staliunas, and C.O. Weiss, Spatial localized structures in resonators with saturable absorber, Opt. Commun. 149, 113-116 (1998).

[59] M. Tlidi, P. Mandel, and R. Lefever, Localized structures and localized patterns in optical bistability, Phys. Rev. Lett. 73(5), 640-643 (1994).

[60]K. Staliunas and V.J. Sánchez-Morcillo, Localized structures in degenerate optical parametric oscillators, Opt. Commun. 139(4-6), 306-312 (1997).

[61]S. Longhi, Spatial solitary waves and patterns in type II second-harmonic generation, Opt. Lett. 23(5), 346-348 (1998).

[62] D. Michaelis, U. Peschel, and F. Lederer, Multistable localized structures and superlattices in semiconductor optical resonators, Phys. Rev. A 56(5), R3366-R3369 (1997).

[63] L. Spinelli, G. Tissoni, M. Brambilla, F. Prati, and L.A. Lugiato, Spatial solitons in semiconductor microcavities, Phys. Rev. A 58(3), 2542-2559 (1998).

[64] V.B. Taranenko, I. Ganne, R.J. Kuszelewicz, and C.O. Weiss, Patterns and localized structures in bistable semiconductor resonators, Phys. Rev. A 61(6), 063818 (2000).

[65]K. Staliunas and V.J. Sánchez-Morcillo, Spatiallocalized structures in degenerate optical parametric oscillators, Phys. Rev. A 57(2), 1454 (1998).

[66] M. Tlidi, P. Mandel, M. Le Berre, E. Ressayre, A. Tallet, and L. Di Menza, Phase-separation dynamics of circular domain walls in the degenerate optical parametric oscillator, Opt. Lett. 25(7), 487-489 (2000).

[67]G. Izús, M. San Miguel, and M. Santagiustina, Phase-locked spatial domains and Bloch domain walls in type-II optical parametric oscillators, Phys. Rev. E 64(5), 056231 (2001).

[68] K. Staliunas, Three-dimensional turing structures and spatial solitons in optical parametric oscillators, Phys. Rev. Lett. 81(1), 81-84 (1998).

[69] M. Tlidi, M. Haelterman, and P. Mandel, Threedimensional structures in diffractive and dispersive nonlinear ring cavities, Quantum Semiclass. Opt. 10(6), 869 (1998). 
[70]G.J. de Valcárcel and K. Staliunas, Excitation of phase patterns and spatial solitons via twofrequency forcing of a 1:1 resonance, Phys. Rev. E 67(2), 026604 (2003).

[71] G.J. de Valcárcel and K. Staliunas, Pattern formation through phase bistability in oscillatory systems with space-modulated forcing, Phys. Rev. Lett. 105(5), 054101 (2010).

[72] K. Staliunas, G. Slekys, and C.O. Weiss, Nonlinear pattern formation in active optical systems: shocks, domains of tilted waves, and cross-roll patterns, Phys. Rev. Lett. 79(14), 2658 (1997).
[73] K. Staliunas, Midband dissipative spatial solitons, Phys. Rev. Lett. 91(5), 053901 (2003).

[74] K. Staliunas, Midband solitons in nonlinear photonic crystal resonators, Phys. Rev. E 70(1), 016602 (2004).

[75] K. Staliunas and M. Tlidi, Hyperbolic transverse patterns in nonlinear optical resonators, Phys. Rev. Lett. 94(13), 133902 (2005).

[76] S. Kolpakov, A. Esteban-Martín, F. Silva, J. García, K. Staliunas, and G.J. de Valcárcel, Experimental demonstration of hyperbolic patterns, Phys. Rev. Lett. 101(25), 254101 (2008).

\title{
FOTONINIAIS KRISTALAIS UŽPILDYTI REZONATORIAI
}

\author{
M. Peckus a,b, R. Rogalskis a , V. Sirutkaitis ${ }^{\text {a }}$, K. Staliūnas ${ }^{\text {c,d }}$ \\ ${ }^{a}$ Vilniaus universiteto Lazeriniu tyrimu centras, Vilnius, Lietuva \\ ${ }^{\mathrm{b}}$ Fiziniu ir technologijos mokslu centras, Vilnius, Lietuva \\ ${ }^{c}$ Katalonu politechnikos universiteto Fizikos ir branduolinès inžinerijos katedra, Terasa, Ispanija \\ ${ }^{\mathrm{d}}$ Katalonų tyrimų ir aukštuju studiju institutas (ICREA), Barselona, Ispanija
}

\section{Santrauka}

Darbe tiriami plokščiųjų veidrodžių Fabri ir Pero rezonatoriai su vidine lūžio rodiklio moduliacija, atitinkančia vieną išilginị fotoninio kristalo periodą. Tokie rezonatoriai realizuojami sukuriant periodinę lūžio rodiklio moduliaciją vieno arba abiejų veidrodžių paviršiuje (t. y. suformuojant vienmates arba dvimates fazines difrakcines gardeles). Fotoniniu kristalų rezonatoriaus formuojamu pluoštu difrakcinès savybès gali būti apibūdinamos subdifrakciniu (kai kampinis pralaidumo spektras platesnis už homogeninio rezonatoriaus) ir superdifrakciniu (kai kampinis pralaidumo spektras siauresnis už homogeninio rezonatoriaus) režimais. Rezonatorius su vienmate veidrodžių moduliacija išsiskiria hiperboli- nio pavidalo erdviniu spektru, o dvimatès veidrodžių moduliacijos atveju - kvadratinio pavidalo erdviniu spektru. Rezonatoriaus analizei sukurtas sklaidos matricu teorija paremtas modelis. Analizuojamas pagrindinių rezonatoriaus parametrų (veidrodžių pralaidumo, gardeliu difrakcinio efektyvumo, rezonatoriaus ilgio) itaka rezonatoriaus erdvinès dispersijos charakteristikoms. Skirtingai nuo ankstesniu darbų ([20, 21]), šiame darbe pagrindinis demesys skiriamas rezonatoriui su vienu moduliuotu veidrodžiu. Tokio rezonatoriaus eksperimentiškai išmatuoti erdviniai skirstiniai labai gerai atitinka teoriškai sumodeliuotus, o sistema yra patogi netiesiniams šviesos dariniams fotoninių kristalų rezonatoriuose tirti. 